\title{
El oxígeno en la zona radical y su efecto en las plantas
}

\author{
Mario de Jesús Moreno Roblero ${ }^{1}$ \\ Joel Pineda Pineda ${ }^{2 \S}$ \\ Ma. Teresa Colinas León ${ }^{1}$ \\ Jaime Sahagún Castellanos ${ }^{1}$ \\ ${ }^{1}$ Instituto de Horticultura-Universidad Autónoma Chapingo. Carretera México-Texcoco km 38.5, Chapingo, \\ México. CP. 56230. (jaguar_moreno01@ hotmail.com; lozcol@gmail.com). 2Departamento de Suelos- \\ Universidad Autónoma Chapingo. Carretera México-Texcoco km 38.5, Chapingo, México. CP. 56230. \\ ${ }^{\S}$ Autor para correspondencia: pinedapjoel@yahoo.com.mx.
}

\section{Resumen}

La información presentada analiza el estado del arte de las evidencias en los últimos 20 años de la importancia y los efectos de la disponibilidad de oxígeno en la rizósfera sobre el crecimiento y desarrollo de las plantas. Las condiciones adversas de factores bióticos (microrganismos) y abióticos (temperatura, anegamiento, salinidad, otros) que influyen en la concentración de oxígeno en la rizósfera, pueden conducir a una caída rápida en los niveles de oxígeno y afectar la morfología, metabolismo y fisiología de las raíces, con un efecto negativo sobre el crecimiento de la planta. Las técnicas de oxigenación en el medio radical pueden ser favorables, tanto en suelos como en sustratos, en situaciones donde existe un riesgo de hipoxia, pero se requieren estudios específicos sobre el efecto de la aplicación de oxígeno en cada especie, así como la medición de las respuestas para identificar los requerimientos de oxígeno que maximicen el rendimiento y la calidad de los productos cosechados, tanto en campo como en invernadero. En el sector agrícola, se requieren técnicas que sean sustentable y económicamente viables aun con los altos costos que implica el uso de las nuevas tecnologías.

Palabras clave: anoxia, hipoxia, oxidación.

Recibido: febrero de 2020

Aceptado: marzo de 2020 
El oxígeno en el medio de crecimiento juega un papel crítico, ya que determina la orientación radicular y el estado metabólico de la raíz. El oxitropismo permite a las raíces evitar las zonas del sustrato con bajos niveles de oxígeno y podría ser también un mecanismo fisiológico para reducir la competencia entre las raíces por agua, nutrientes y el propio oxígeno (Porterfield and Musgrave, 1998).

La disponibilidad de oxígeno a nivel radical es fundamental para el desarrollo óptimo de las plantas, ya que se requiere en diferentes procesos metabólicos prioritarios como: el metabolismo de carbohidratos, la reducción de nitratos, la fijación simbiótica de nitrógeno, la renovación de proteínas, el mantenimiento del gradiente de protones y la absorción de nutrientes por las raíces (M'Bou et al., 2010; Makita et al., 2015; Bhatla, 2018), con efectos inmediatos sobre el crecimiento radical y el vástago, lo que repercute en el desarrollo general de la planta (Morard et al., 2000; Dutilleul et al., 2003; Eckhard et al., 2013).

La respiración en las plantas es un proceso oxidativo controlado por diferentes vías metabólicas, con el fin de evitar situaciones de baja disponibilidad de energía (Toro y Pinto, 2015) aún en situaciones de hipoxia (van Dongen et al., 2011; Gupta et al., 2015), aunque el conocimiento de su regulación es limitado (Fernie et al., 2004; Ishizawa, 2014). Existen deficiencias frecuentes de oxígeno en la rizósfera, como resultado de altas tasas de respiración radical, ya sea por temperaturas elevadas, por deficiencias en la capacidad de aireación del medio de cultivo o por un manejo inadecuado del riego (Carazo et al., 2015).

Esto ha dado lugar a diferentes prácticas culturales y técnicas de oxigenación (aeroponia, oxidación, oxifertirrigación) del medio radical, del agua de riego o de la solución nutritiva (Marfá et al., 2005; Bhattarai et al., 2006; Midmore, 2007). Con base en lo anterior, el objetivo de este trabajo es revisar las evidencias sobre los efectos y la importancia de la disponibilidad de oxígeno en la rizósfera para el crecimiento y desarrollo de las plantas, así como los beneficios de la oxifertirrigación al minimizar el riesgo de hipoxia e incrementar rendimiento y calidad de los productos cosechados en los cultivos agrícolas.

\section{Requerimientos de oxígeno en las plantas}

Es difícil definir con valores de concentraciones específicas la condición de disponibilidad de oxígeno para las plantas. La terminología para describir esa condición se basa en la comparación con el nivel de oxígeno atmosférico $(21 \% \mathrm{v} / \mathrm{v})$, conocida como normoxia. Las concentraciones de oxígeno mayores o menores se denominan superoxia e hipoxia, respectivamente; mientras que la anoxia es la situación en la que no hay oxígeno libre disponible.

La anoxia también se utiliza para describir las concentraciones de oxígeno dentro del tejido que están muy cerca de cero. La interpretación de la hipoxia es complicada porque se utiliza tanto para referirse a la concentración de $\mathrm{O}_{2}$ en el ambiente como su concentración dentro del tejido vegetal; por ejemplo, un tejido que se mantiene en un ambiente normoxico puede ser hipóxico en el interior; por ello, se debe especificar la concentración $\mathrm{O}_{2}$ en cada situación (Fagerstedt et al., 2013; van Dongen y Licausi, 2015). 
La respiración de las raíces en condiciones aeróbicas, requiere un suministro continuo de $\mathrm{O}_{2}$ a la rizósfera. La respiración radical depende de diversos factores: temperatura (Rachmilevitch et al., 2006), salinidad (Bernstein et al., 2013), metales pesados, estrés hídrico (Jiménez et al., 2013) y saturación del espacio poroso (Liao y Lin, 2001), pero el factor que tiene la relación más directa es la disponibilidad de $\mathrm{O}_{2}$, que permite o no el proceso, convirtiéndolo en un factor limitante tanto en suelo como en sustratos (Urrestarazu y Mazuela, 2005; Fagerstedt et al., 2013).

Como resultado, las plantas pueden exhibir disminución en consumo de agua, marchitamiento y conductancia estomática reducida (Bhatla, 2018), crecimiento lento y disminución del rendimiento (Bhattarai et al., 2008; Maestre y Martínez, 2010). El daño y la muerte de las plantas inundadas se han atribuido a la falta de oxígeno en la raíz (Boru et al., 2003; Bhatla, 2018).

Por el papel central en el metabolismo energético (respiración), metabolismo del N, sustrato en reacciones de desaturación de ácidos grasos y síntesis de fitohormonas como el etileno, el ácido abscísico y las giberelinas (van Dongen y Licausi, 2015). En condiciones de hipoxia, además de las propiedades físicas que restringen la disponibilidad de $\mathrm{O}_{2}$ (Friedman y Naftaliev, 2012), también la actividad respiratoria de los microorganismos disminuye su contenido, ya que compiten con la planta por el $\mathrm{O}_{2}$ disponible, sobre todo en sustratos orgánicos inestables o inmaduros con actividad microbiana alta (Lemaire, 2005). También el bajo contenido de $\mathrm{O}_{2}$ aumenta la susceptibilidad a la infección por patógenos, ya que las esporas se estimulan con los exudados en la rizósfera, como es el caso de Phytophthora infestans, donde el anegamiento genera respiración anaeróbica con liberación de etanol que estimula la producción de estructuras de infección (Lal et al., 2018).

Aunque los efectos graves que tiene la anoxia en la planta (Gibbs y Greenway, 2003) son poco probables en los sustratos porosos utilizados en los sistemas de cultivo sin suelo (Morard et al., 2000) y las plantas exhiben tolerancia a la deficiencia de $\mathrm{O}_{2}$ durante varias horas (Geigenberger, 2003), ya que cuando estas condiciones se revierten las plantas no sufren efectos secundarios, se han observado respuestas positivas a la oxigenación en condiciones de hipoxia y anoxia en estudios con cultivos en solución nutritiva (Marfá et al., 2005; Urrestarazu y Mazuela, 2005; Urrestarazu et al., 2006).

En raíces de maíz (Zea mays L.) se observó un gradiente de 10 a 5\% de $\mathrm{O}_{2}$ desde el córtex hasta la estela. En higuerilla (Ricinus communis L.) el gradiente se estableció entre $21 \%$ del exterior a $7 \%$ en el floema. En raíces de chícharo (Pisum sativum) se midió una concentración interna de 8\% de $\mathrm{O}_{2}$ y en tubérculo de papa (Solanum tuberosum L.) el gradiente de oxígeno se estableció de 1115\% del peridermo a 2-5\% de oxígeno en el centro (Papke et al., 2014).

\section{El oxígeno en el agua}

La solubilidad del $\mathrm{O}_{2}$ en el agua depende de la temperatura, la presión parcial de $\mathrm{O}_{2}$, la presión atmosférica, la salinidad del agua y el área de contacto entre agua y aire. Pero en condiciones normales $\left(20{ }^{\circ} \mathrm{C}, 1\right.$ atmósfera de presión, 20-21\% de $\mathrm{O}_{2}$ en el aire), la cantidad máxima en solución está entre 8 y $9 \mathrm{mg} \mathrm{L}^{-1}$. Esta concentración disminuye a medida que aumenta la temperatura y la concentración de sales disueltas (Cuadro 1) (Murad et al., 2005; Massol, 2005). 
Cuadro 1. Solubilidad del oxígeno $\left(\mathrm{mg} \mathrm{L}^{-1}\right)$ en función de la temperatura y la salinidad (Massol, 2005).

\begin{tabular}{ccccccc}
\hline \multirow{2}{*}{ Temperatura $\left({ }^{\circ} \mathrm{C}\right)$} & \multicolumn{5}{c}{ Salinidad $(\%)$ de saturación en la solución } \\
\cline { 2 - 6 } & 0.03 & 9.055 & 18.08 & 27.105 & 36.13 & 45.155 \\
\hline 0 & 14.624 & 13.728 & 12.888 & 12.097 & 11.355 & 10.657 \\
5 & 12.77 & 12.024 & 11.32 & 10.656 & 10.031 & 9.441 \\
10 & 11.288 & 10.656 & 10.058 & 9.493 & 8.959 & 8.454 \\
15 & 10.084 & 9.541 & 9.027 & 8.54 & 8.079 & 7.642 \\
20 & 9.092 & 8.621 & 8.174 & 7.749 & 7.346 & 6.964 \\
25 & 8.263 & 7.85 & 7.457 & 7.083 & 6.728 & 6.39 \\
30 & 7.559 & 7.194 & 6.845 & 6.513 & 6.1 & 5.806 \\
35 & 6.95 & 6.624 & 6.314 & 6.017 & 5.734 & 5.464 \\
40 & 6.412 & 6.121 & 5.842 & 5.576 & 5.321 & 5.078 \\
45 & 5.927 & 5.665 & 5.414 & 5.174 & 4.944 & 4.724 \\
50 & 5.477 & 5.242 & 5.016 & 4.799 & 4.591 & 4.392 \\
\hline
\end{tabular}

La concentración de $\mathrm{O}_{2}$ disuelto (COD) es un parámetro importante de la calidad del agua de riego que puede convertirse en un factor limitante en algunos sistemas agrícolas intensivos (Raviv et al., 2004; Marfá et al., 2005). En hidroponia, Morard (1995) estableció la presión parcial crítica de $\mathrm{O}_{2}$ en 4-6\% en la solución nutritiva, que corresponde a valores de COD aproximadamente $3 \mathrm{mg} \mathrm{L}^{-1}$.

\section{El oxígeno en el suelo}

La porosidad de aire en el suelo está determinada por la textura, el tamaño de agregado y el grado de compactación. Las partículas más pequeñas (limo y arcilla) reducen la aireación porque se unen muy estrechamente, limitando directa e indirectamente los espacios de aire entre ellas, ya que retienen más agua contra las fuerzas de drenaje (Rengasamy, 2000).

Las partículas más grandes (arena), los agregados y la materia orgánica aumentan la aireación porque dejan poros que drenan y se llenan fácilmente por aire. En suelos arenosos a capacidad de campo (CC), el aire ocupa $25 \%$ o más de su volumen, pero a mayor aireación, la evaporación y la lixiviación se incrementan rápidamente. En suelos arcillosos, el volumen de aire a CC está entre 15 y $20 \%$, pero puede ser menor a $10 \%$ (Peverill, 1999).

Por diferencias de presión, el oxígeno de la atmósfera se difunde a través de los poros llenos de aire hasta la cercanía de las raíces, ya que su difusión; a través, de los poros con agua es más lenta. Luego se disuelve en la película de agua que rodea a las raíces y por difusión se introduce en las células donde la respiración y generará energía para otros procesos metabólicos (Bonachela et al., 2008). 


\section{El oxígeno en los sustratos}

Elevada disponibilidad de agua, adecuado suministro de aire, baja densidad aparente y alta estabilidad estructural son las principales características físicas del sustrato ideal, en el cual, la tasa de difusión de oxígeno (TDO) está relacionada con la capacidad de aireación, cuyos valores mayores a 30\% no tendrán TDO limitantes para la absorción de agua y nutrientes (Bonachela et al., 2008; Pineda et al., 2012).

Las plantas que crecen en contenedores, especialmente las que duran confinadas por largos periodos, desarrollan mayor cantidad de raíces en el fondo y en el espacio entre el sustrato y las paredes del contenedor (Gallegos et al., 2020). Esto se debe a que la compactación produce deficiencia de oxígeno y muerte de las raíces en el centro del contenedor, fenómeno que es más acentuado en sustratos compactados que presentan resistencia mecánica a la penetración radical y en sustratos con contenido alto de materia orgánica que entran en descomposición por microorganismos consumidores de oxígeno. El crecimiento vertical hacia abajo es una respuesta natural al gravitropismo e hidrotropismo, típico de todas las raíces activas.

Sin embargo, en contenedores, esto resulta en una maraña de raíces en el fondo del contenedor expuestas a deficiencia de oxígeno debido a la competencia entre ellas agravada con las frecuentes acumulaciones de agua (Lemaire, 2005; Pineda et al., 2012). En contenedores, las arenas tienden a compactarse debido a la manipulación, los impactos físicos en la superficie del sustrato cuando se mueven los contenedores y la sobre irrigación generan compactación, proceso que es irreversible, reduciéndose el crecimiento de la raíz (Kafkafi, 2008).

Las consecuencias más comunes son: a) acumulación salina cuando la zona radicular no es lo suficientemente lavada por el agua de riego; b) toxicidad de amonio debido a altas concentraciones de fertilizantes durante periodos de alta temperatura; y c) sobrecalentamiento por exposición de los contenedores a la radiación directa del sol y en consecuencia muerte de raíces debido a deficiencia de oxígeno que se acentúa con el riego excesivo, especialmente durante la estación caliente (Lemaire, 2005; Kafkafi, 2008).

La saturación del sustrato provoca una disminución en el intercambio gaseoso entre la atmósfera y la rizósfera, en consecuencia, la concentración de $\mathrm{O}_{2}$ para la respiración radical será un factor limitante (Raviv et al., 2004). Aun en sustratos bien aireados pueden ocurrir deficiencias de $\mathrm{O}_{2}$ en períodos o áreas donde las altas tasas de crecimiento y respiración de la raíz coinciden con altas temperaturas y una disminución en la concentración de $\mathrm{O}_{2}$ disuelto en la solución del sustrato (Raviv et al., 2004; Bonachela et al., 2007; Acuña et al., 2008).

\section{Mecanismos de tolerancia a la deficiencia de oxígeno}

En suelos y sustratos con deficiencia de $\mathrm{O}_{2}$ las plantas se adaptan modificando sus características morfológicas, anatómicas, fisiológicas y metabólicas, dependiendo del grado de estrés por $\mathrm{O}_{2}$ (Cuadro 2) y varían ampliamente entre especies de plantas y entre genotipos de la misma especie (Fagerstedt et al., 2013; Larsen et al., 2015; Bhatla, 2018). 
Cuadro 2. Efectos negativos y modificaciones en las plantas bajo condiciones de hipoxia.

Estrés por la deficiencia de $\mathrm{O}_{2} \quad$ Mecanismo de ajuste o adaptación por la planta

Alta sensibilidad a la falta de $\mathrm{O}_{2}$ en tejido interno

Disminución de potencial redox (Eh) y formación de especies químicas reducidas toxicas $\left(\mathrm{Mn}^{2+}, \mathrm{Fe}^{2+}, \mathrm{H}_{2} \mathrm{~S}\right)$ y perdidas de $\mathrm{NO}_{3}{ }^{-}$por desnitrificación

Alteración en metabolismo de carbohidratos (almidones y sacarosa) en la respiración aeróbica como proceso central precursor de sustratos y ATP para diferentes reacciones del metabolismo primario y secundario (crisis energética)

Acumulación de oxidantes y radicales libres $\mathrm{ROS}\left(\mathrm{O}_{2}^{-}, \mathrm{HO}^{-}, \mathrm{HO}_{2}^{-}\right.$, $\mathrm{H}_{2} \mathrm{O}_{2}, \mathrm{NO}, \mathrm{ONOO}^{-}$)

Generación de acidosis citosólica

Acumulación de toxinas: ácido láctico, etanol, acetaldehído Desbalance en fitohormonas: incremento de ACC/etileno en raíz y disminución de citoquininas y giberelinas en parte aérea.
Modificaciones morfo-anatómicas:

Alargamiento de tallo y peciolos de hoja hacia la superficie del agua (zonas inundadas)

Adelgazamiento de las hojas para facilitar la fotosíntesis bajo el agua

Formación de aerénquima para transportar $\mathrm{O}_{2}$ desde las estructuras aéreas hasta las raíces sumergidas Formación de raíces adventicias, que desarrollan aerénquima para transporte de $\mathrm{O}_{2}$ del exterior hacia el tejido deficiente de $\mathrm{O}_{2}$

Lenticelas para el intercambio gaseoso entre exterior e interior de los tejidos

Desarrollo de barreras contra solutos y gases (suberización en exodermis y lignificación en células esclerénquima, mayor espesor de hipodermis) para disminuir la pérdida de $\mathrm{O}_{2}$ suministrado por aerénquima y evitar la entrada de especies químicas reducidas tóxicas $\left(\mathrm{Mn}^{2+}, \mathrm{Fe}^{2+}, \mathrm{H}_{2} \mathrm{~S}\right)$

Menor espesor de estela para minimizar la demanda de $\mathrm{O}_{2}$ en el centro de la raíz

Raíces poco profundas para evitar las zonas con menores potenciales redox (Eh)

Ajustes y modificaciones fisiológicas y metabólicas:

El metabolismo de carbohidratos (almidones y sacarosa) en la respiración como proceso central precursor de sustratos para diferentes reacciones del metabolismo primario y secundario El metabolismo energético del ATP para minimizar sus requerimientos en diferentes procesos

Producción de antioxidantes (SOD-Fe, SOD-Mn, SODZn. SOD-Cu, GSH/GSSG) para la detoxificación de radicales libres ROS

Ajuste en metabolismo del $\mathrm{N}$ para estabilización de $\mathrm{pH}$ citosólico (homeostasis)

Evitar acumulación de los productos finales (lactato, etanol) con metabolismo interno y extrusión al exterior

Regulación en la relación parte aérea/raíz para aumentar eficiencia en absorción de agua, nutrientes y transporte de hormonas.

Incremento en el suministro de $\mathrm{O}_{2}$ a través del aerénquima

Adaptado de Eckhard et al. (2013); Fargestedt et al. (2013); Ishizawa (2014); Limami (2014); Larsen et al. (2015); Bhatla (2018). 
La tolerancia de las plantas a la falta de $\mathrm{O}_{2}$ depende de su capacidad para mitigar los efectos perjudiciales de la crisis energética y la acidosis citosólica. La fermentación láctica y etanólica se activan bajo estrés de $\mathrm{O}_{2}$ con el fin de regenerar $\mathrm{NAD}^{+}$para mantener una alta tasa de glucólisis que se convierte en la principal vía para la producción de ATP. Paradójicamente el ácido láctico empeora la acidosis citosólica y se consume carbono para la formación de etanol, un producto sin salida metabólicamente inútil.

Esta adaptación ahorra ATP, regenera $\mathrm{NAD}^{+}$y ahorra carbono en forma de alanina, una forma de almacenamiento $\mathrm{C} / \mathrm{N}$ removilizable después de su formación. La acumulación de alanina contribuye indirectamente a la homeostasis del $\mathrm{pH}$ mediante el consumo de ácido pirúvico y compitiendo con lactato deshidrogenasa (Eckhard, 2013; Limami, 2014).

Al respecto, Ishizawa (2014) reportó que en maíz el pH citoplásmico de células muertas disminuyó de 7.5 a 6.2, pero permaneció en 6.8 después de 12 h de hipoxia en raíces normales, demostrando que la acidosis citoplasmática altera la homeostasis del $\mathrm{pH}$ en las células, lo que conduce a la muerte celular en condiciones de hipoxia y anoxia.

\section{Enriquecimiento de oxígeno en la rizósfera}

Se han probado varios métodos para mejorar el suministro de $\mathrm{O}_{2}$ a las raíces de los cultivos; en suelos se debe mejorar la estructura a través de la labranza (Abu-Amdeh, 2003), crear vías de aireación artificiales, establecer drenaje y seleccionar plantas tolerantes (Stepanova et al., 2002). La deficiencia de $\mathrm{O}_{2}$ puede complicarse aún más cuando se usan aguas residuales, ya que el contenido de $\mathrm{O}_{2}$ disuelto en esta agua es bajo o cercano a cero, especialmente cuando se almacena en tanques cerrados (Bonachela et al., 2007), además esta agua generalmente tiene un alto contenido de materia orgánica que conduce a una mayor actividad microbiana dentro del suelo, lo que aumenta la competencia por el oxígeno en el entorno de la raíz (Bonachela et al., 2008). La baja concentración de $\mathrm{O}_{2}$ en el agua de riego puede evitarse usando varios métodos de enriquecimiento de $\mathrm{O}_{2}$.

La oxifertirrigación se ha desarrollado para mejorar la disponibilidad de oxígeno de la rizósfera y consiste en suministrar $\mathrm{O}_{2}$ disuelto en el agua de riego a concentraciones sobresaturadas usando equipo de fertirrigación (Marfá et al., 2005). La información sobre la dinámica del contenido de oxígeno en la solución del sustrato en cultivos de hortalizas en invernadero es escasa (Bonachela et al., 2007) y la mayoría de los estudios se han llevado a cabo en períodos cortos de cultivo en condiciones controladas de clima (Shi et al., 2007).

Se inyecta aire presurizado en el sistema de riego por goteo (Bhattarai et al., 2006; Bonachela et al., 2010; Maestre y Martínez, 2010) o gas oxígeno a la solución nutritiva (Marfá et al., 2005; Urrestarazu y Mazuela, 2005), también se añade ácido peroxiacético $\left(\mathrm{CH}_{3} \mathrm{CO}_{3} \mathrm{H}\right)$ (Carrasco et al., 2010), peróxido de hidrógeno $\left(\mathrm{H}_{2} \mathrm{O}_{2}\right)$, peróxido de urea $\left(\mathrm{CH}_{6} \mathrm{~N}_{2} \mathrm{O}_{3}\right)$, peróxido de calcio $\left(\mathrm{CaO}_{2}\right)$, peróxido de potasio $\left(\mathrm{K}_{2} \mathrm{O}_{2}\right)$ u ozono $\left(\mathrm{O}_{3}\right)$ al agua de riego (Urrestarazu y Mazuela, 2005; Mazuela, 2010), estos compuestos se consideran como fertilizantes de oxígeno (Mazuela, 2010).

La mayoría de los métodos buscan mantener las concentraciones de oxígeno disuelto arriba de 3 $4 \mathrm{mg} \mathrm{L}{ }^{-1}$, para evitar una disminución en el crecimiento radical y un pardeamiento de las raíces (Urrestarazu et al., 2006). En la práctica, la oxigenación del suelo o sustrato es compleja ya que 
hay otros factores que pueden afectar tanto la demanda como al suministro de $\mathrm{O}_{2}$ y provocar su deficiencia o hipoxia (presión parcial de oxígeno entre 1 y 4\%), condición que reduce la actividad de la planta (Urrestarazu et al., 2006).

La mayoría de los cultivos hortícolas cultivados en sustrato y en invernadero tienen altas tasas de crecimiento asociadas a altas tasas de respiración y demanda de agua, forman una densidad alta de raíces en el fondo del contenedor, donde suelen ocurrir condiciones cercanas a saturación. El intercambio gaseoso dentro del sustrato puede disminuir por el contenedor (normalmente plástico) que rodea al sustrato.

\section{Oxigenación y su influencia en los cultivos}

Aunque se han reportado algunos resultados contradictorios respecto al uso de métodos para enriquecer con oxígeno el medio de crecimiento de los cultivos, especialmente con condiciones de ambiente no controlado (Urrestarazu y Mazuela, 2005; Bonachela et al., 2007), diversos experimentos con producción de plantas bajo invernadero, ya sea en solución nutritiva o en sustrato, indican que altos niveles de oxigenación aceleran el desarrollo e incrementan los rendimientos, como resultado de la mejora en procesos anatómico-fisiológicos e incremento en la conductividad hidráulica de las raíces, mayor absorción de minerales y fotosíntesis más eficiente (Urrestarazu et al., 2006).

Morard et al. (2000) reportaron en melón (Cucumis melo L.), con un desarrollo radical de $25 \mathrm{~g} \mathrm{~L}^{-1}$ y solución nutritiva a $20{ }^{\circ} \mathrm{C}$, que la concentración a saturación de $8 \mathrm{mg} \mathrm{L}^{-1}$ se reduce a $0 \mathrm{mg} \mathrm{L}^{-1}$ en 60 min, si no hay reposición de oxígeno. El suministro de oxígeno en rosa (Rosa sp.) incrementó la actividad de la enzima alcohol deshidrogenasa (ADH), mejoró el crecimiento y desarrollo de raíces y tallos en jitomate (Solanum lycopersicum L.).

Experimentos de Goorahoo et al. (2002) con cinta de goteo a $12-15 \mathrm{~cm}$ de profundidad y estudios de Bhattarai et al. (2006) con cinta a 8 a $30 \mathrm{~cm}$ de profundidad, mostraron los beneficios de la oxigenación. Los datos mostraron que el incremento en rendimiento de fruto de pimiento (Capsicum annuиm L.) alcanzó su valor máximo a $25 \mathrm{~m}$ de la fuente de aireación y luego disminuyó a lo largo de $35 \mathrm{~m}$, igualando al final de la fila el rendimiento de las parcelas sin aireación.

En ensayos de oxigenación con inyección de aire con venturi y peróxido de hidrógeno en un suelo arcilloso cultivado con jitomate, soya (Glycine max L.) y algodón (Gosipium irsutum L.), se obtuvieron incrementos en rendimiento de 12,84 y $21 \%$, respectivamente, en comparación con el testigo (Bhattarai et al., 2006). De manera similar en un suelo arcilloso y franco arenoso, la oxigenación con venturi produjo un aumento de 33 y 39\%, respectivamente, en el peso total del fruto de pimiento (Goorahoo et al., 2002).

La soya se adaptó a deficiencia prolongadas de oxígeno produciendo raíces adventicias, el tallo manifestó hipertrofia y desarrolló aerénquima para el transporte de $\mathrm{O}_{2}$ a las raíces (Boru et al., 2003). La eficiencia del uso del agua (EUA) con oxigenación fue $11 \%$ mayor (39.1 versus $35.2 \mathrm{~g}$

$\left.\mathrm{L}^{-1}\right)$ para jitomate, $70 \%$ (3.65 versus $\left.2.15 \mathrm{~g} \mathrm{~L}^{-1}\right)$ para soya y $18 \%\left(0.45\right.$ versus $\left.0.38 \mathrm{~g} \mathrm{~L}^{-1}\right)$ para algodón en un suelo arcilloso (Bhattarai et al., 2006) y 36\% (1.46 versus $0.94 \mathrm{~kg} \mathrm{~m}^{-3}$ ) para maíz en un suelo franco arcillo-arenoso (Abuarab et al., 2012). 
Otros efectos de EUA, como la relación fotosíntesis/transpiración neta de la hoja, también fueron mayores con la oxigenación (Bhattarai et al., 2006). Urrestarazu y Mazuela (2005) suministraron $\mathrm{O}_{2}$ con peróxido de potasio $\left(1 \mathrm{~g} \mathrm{~L}^{-1}\right)$ a pimiento y melón, encontrando un rendimiento de 20 y $15 \%$ más alto, respectivamente, con oxigenación; pero no hubo diferencias significativas en pepino.

Al respecto, Urrestarazu et al. (2006) indican que la aplicación de un oxigenante químico a la solución de riego incrementa significativamente la disponibilidad de $\mathrm{O}_{2}$, traduciéndose en mayor rendimiento de frutos por planta. El riego subterráneo con $12 \%$ de aire en el agua, estimuló el crecimiento y mejoró el rendimiento reproductivo al adelantar la floración y fructificación en jitomate, e incrementó el rendimiento en $21 \%$, el efecto de la aireación fue mayor en suelo a capacidad de campo (CC) en relación al suelo con estrés hídrico; mientras que en suelo salino, el incremento fue $38 \%$ en relación al suelo sin aireación.

En ambos casos, aumentó la eficiencia del uso del agua, 16\% en los experimentos de humedad y $32 \%$ en salinidad. El aumento en el rendimiento con aireación también fue acompañado con mayor índice de cosecha (IC), mayor peso de los frutos, y un menor índice de estrés hídrico. El beneficio obtenido por la oxigenación del agua de riego no sólo se observó en condiciones en las que la porosidad de aire era baja (suelos sódicos de mala estructura o a CC en suelos arcillosos), sino también en suelos con menor disponibilidad de humedad.

El riego a $\mathrm{CC}$ en el suelo arcilloso generó menor concentración de $\mathrm{O}_{2}$ en la rizósfera, pero la aireación mejoró la concentración de $\mathrm{O}_{2}$ disuelto e incrementó el rendimiento de jitomate, ya que la aireación evitó la hipoxia (Bhattarai et al., 2006). Otro estudio de Bonachela et al. (2007) demostró que la oxigenación de la solución del sustrato en cultivo de pimiento y jitomate evitó condiciones de hipoxia, mientras que en melón ocurrieron deficiencias de $\mathrm{O}_{2}$ durante la segunda mitad del ciclo de cultivo, cuando se presentaron altas temperaturas ambientales y la planta había desarrollado su sistema radical y dosel completos.

En plantas de rosa que recibieron tratamientos de oxifertirrigación, el área foliar, el peso seco de hojas y flores fueron mayores, en aquellas que crecieron en sustratos de partícula fina con presencia de hipoxia (Carazo et al., 2008). En estos periodos, enriquecer con $\mathrm{O}_{2}$ la solución nutritiva aportada, mediante las balsas de riego o aportes artificiales de $\mathrm{O}_{2}$ puede mejorar las condiciones de crecimiento de los cultivos y en consecuencia su productividad (Bonachela et al., 2008).

En el cultivo de rúcula (Eruca sativa Mill), se evaluaron diferentes dosis de ácido peroxiacético $\left(\mathrm{CH}_{3} \mathrm{CO}_{3} \mathrm{H}\right)$ en la solución nutritiva, determinándose que $40 \mathrm{mg} \mathrm{L}^{-1}$ produjeron mayor rendimiento en comparación con plantas cultivadas en perlita sin adición de este ácido (Carrasco et al., 2010). En los parámetros de fertirrigación, producción y calidad de frutos de melón, la aplicación del oxigenante peróxido de potasio incrementó la absorción y la eficiencia de uso del agua en términos de kilogramos producidos por litro de agua consumida.

No hubo diferencias en el rendimiento y la calidad de la fruta, lo que se atribuyó a la ausencia de deficiencia de $\mathrm{O}_{2}$ en el tratamiento control y a períodos de hipoxia de intensidad o duración insuficiente para afectar significativamente la productividad (Mazuela, 2010). Con la inyección de aire en riego por goteo subsuperficial se incrementó la productividad de papa, el rendimiento fue 27.11 y $17.8 \%$. Asimismo, fue mayor en el tratamiento de inyección de aire, en comparación con el riego por goteo (RG) y el riego por goteo subterráneo (RGS), respectivamente, en el primer ciclo, 
pero fue 38.2 y $7.66 \%$ mayor que RG y RGS, respectivamente, en el segundo ciclo. En el tratamiento de inyección de aire, en comparación con los tratamientos sin aireación (RG) y (RGS), el uso eficiente del agua (UEA) fue 46.41 y 30.52\% mayor en el primer ciclo, y resultó 61.78 y $19.33 \%$ mayor que en RG y RGS, respectivamente, para el segundo ciclo.

La altura de planta fue 14.7 y $6.07 \%$ mayor en el tratamiento de inyección de aire que en el control (RG) y (RGS), respectivamente, para el primer ciclo, mientras que fue 14.13 y $9.7 \%$ mayor que en los controles en el segundo ciclo. El peso de los brotes por planta fue 14.8 y $4.61 \%$ mayor en el tratamiento con aire que en RG y RGS, respectivamente, para el primer ciclo, mientras que fue mayor en un 37.6 y $1.94 \%$ para el segundo ciclo.

Estos datos indican que el rendimiento de papa puede mejorarse en riego por goteo subterráneo si el agua es aireada (Mohamed et al., 2014). Carazo (2015) reportó que la oxifertirrigación favoreció la productividad en rosa (número de flores, número y peso de frutos), la calidad (peso y longitud de la flor cortada, duración de flor cortada, peso y superficie foliar y radicular de esqueje, plántula enraizada) y el desarrollo en diversos aspectos (parámetros biométricos, contenido mineral).

Cuando se inyecta aire en el agua dentro de la zona de las raíces, aumenta la difusión de etileno y dióxido de carbono fuera de la rizósfera, lo que da lugar a mejores condiciones de crecimiento de la planta. El aumento del rendimiento y la mejora potencial de la calidad del suelo asociada con la aireación de la zona de raíces implica la adopción de la tecnología de inyección de aire en el sistema de riego como herramienta para aumentar la productividad de los cultivos (Mohamed et al., 2014).

\section{Conclusiones}

La información presentada resume las evidencias sobre los efectos y la importancia de la disponibilidad de oxígeno en la rizósfera para el crecimiento y desarrollo de las plantas. Hace falta ampliar los estudios sobre los beneficios del suministro constante o intermitente de oxígeno, mediante diferentes métodos, durante las etapas del desarrollo de las plantas o condiciones donde exista una demanda elevada de oxígeno.

Se requieren estudios específicos sobre el efecto del oxígeno en diferentes especies de plantas, así como la medición de las respuestas para identificar los requerimientos de oxígeno que maximicen el rendimiento y la calidad de los productos cosechados en los agrosistemas tanto en campo como en invernadero. Los resultados a la fecha indican, que la oxifertigación puede ser un factor de manejo para aumentar la eficiencia de los recursos naturales utilizados en sistemas intensivos de producción de cultivos agrícolas.

\section{Literatura citada}

Abu-Amdeh, N. H. 2003. Effect of compaction and deep tillage on soil hydraulic and aeration properties and wheat yield. Communications in Soil Science and Plant Analysis. 34:2277-2290.

Abuarab, M. E.; Mostafa, E. and Ibrahim, M. M. 2012. Effect of air injection under subsurface trickle irrigation on yield and water use efficiency of corn in a sandy clay loam soil. Journal of Advanced Research. 
Acuña, R. A.; Gil, I.; Bonachela, S. and Magán, J. J. 2008. Oxyfertigation of a greenhouse melon crop grown in rockwool slabs in a mediterranean area. Acta Horticulturae. 10.17660/ActaHortic.208.779.56.

Bernstein, N.; Eshel, A. and Beeckman, T. 2013. Effects of salinity on root growth, 595-612. In: Eshel A and Beeckman T. (Eds). Plant roots. The hidden half. Fourth edition. CRC Press, $831 \mathrm{p}$.

Bhattarai, S. P.; Pendergast, L. and Midmore, D. J. 2006. Root aeration improves yield and water use efficiency of tomato in heavy clay and saline soils. Scientia Horticulturae. 108:278-288.

Bhattarai, S. P.; Su, N.; Midmore, D. J. and Pendergast, L. 2008. Yield, water-use efficiencies and root distribution of soybean, chickpea and pumpkin under different subsurface drip irrigation depths and oxygation treatments in vertisols. Irrig. Sci. 26:439-450.

Bonachela, S.; Casas, J. J. and Acuña, R. A. 2007. Environmental factors and management practices controlling oxygen dynamics in agricultural irrigation ponds in a semiarid Mediterranean region: implications for pond agricultural functions. Water Res. 41:1225-1234.

Boru, G.; Vantoai, T.; Alves, J.; Hua, D. and Knee, M. 2003. Responses of Soybean to Oxygen Deficiency and Elevated Root-zone Carbon Dioxide Concentration. Annals of Botany. 91:447-453.

Carazo, N.; López, D.; Rodrigo, M. C.; Brunet, G.; Cáceres, R. and Marfà, O. 2008. The effect of oxygenation on the root hydraulic conductivity of container-grown roses. Acta Hortic. 779:471-476.

Carazo, G. N. 2015. Oxifertirrigación en cultivo sin suelo de rosa para flor cortada (Rosa sp.) y pimiento (Capsicum annuum L.): efectos en desarrollo y producción. Tesis Doctoral. Lleida. Lleida, España. 83 p.

Carrasco, G.; Gajardo, J. M.; Álvaro, J. E. and Urrestarazu, M. 2010. Rocket production (Eruca sativa Mill.) in floating system using peracetic acid using as oxygen source compared with substrate culture. J. Plant Nutr. 23:38-46.

Dutilleul, C.; Driscoll, S.; Cornic, G.; De Paepe, R.; Foyer, C. H. and Noctor, G. 2003. Functional mitochondrial complex $\mathrm{I}$ is required by tobacco leaves for optimal photosynthetic performance in photorespiratory conditions and during transients. Plant Physiology, 131(1):264-275.

Eckhard, G.; Horst, J. W. and Neumann, E. 2013. Adaptation of Plants to Adverse Chemical Soil Conditions. 408-471 pp. In: Marschner P. (Ed). Marschner's Mineral Nutrition of Higher Plants. Third Edition. Elsevier. 649 p.

Fagerstedt, V. K.; Blokhina, B. O.; Pucciariello, C. and Perata, P. 2013. Flooding Tolerance Mechanisms in Roots. 498-529 pp. In: Eshel A. and Beeckman T. (Eds). Plant Roots. The hidden half. Fourth edition. CRC Press. 831 p.

Fernie, A. R.; Carrari, F. and Sweetlove, L. J. 2004. Respiratory metabolism: glycolysis, the TCA cycle and mitochondrial electron transport. Current opinion in plant biology. 7(3):254-261.

Friedman, S. P. and Naftaliev, B. 2012. A survey of the aeration status of drip-irrigated orchards. Agricultural Water Management. 115:132-147.

Gallegos, J.; Álvaro E. J. and Urrestarazu M. 2020. Container design affects shoot and root growth of vegetable plant. Hortscience. https://doi.org/10.21273/HORTSCI14954-20.

Geigenberger, P. 2003. Response of plant metabolism to little oxygen. Curr. Opin. Plant Biol. 6:247-256. 
Gibbs, J. and Greenway, H. 2003. Mechanisms of anoxia tolerance in plants. I. Growth, survival and anaerobic catabolism. Funct. Plant Biol. 30:1-47.

Goorahoo, D.; Carstensen, G.; Zoldoske, D. F.; Norum, E. and Mazzei, A. 2002. Using air in subsurface drip irrigation (SDI) to increase yields in bell pepper. In: Proceedings of the Irrigation Association Technical Conference, San Antonio, Texas. 95-102 pp.

Gupta, K. J.; Neelwarne, B. and Mur, L. A. J. 2015. Integrating classical and alternative respiratory pathways, Chap I. In: Gupta K. J., Mur, J. A. L. and Neelwarne, B. (Eds). Alternative Respiratory Pathways in Higher Plants. John Wiley \& Sons Ltd.

Ishizawa, K. 2014. Intracellular pH Regulation of Plant Cells Under Anaerobic Conditions. 59-74 pp. In: van Dongen T. J. and Licausi, F. (Eds). Low-Oxygen Stress in Plants. Oxygen Sensing and Adaptive Responses to Hypoxia. Plant Cell Monographs. Springer. 1250 p.

Jiménez, S.; Dridi, J.; Gutiérrez, D.; Moret, D.; Irigoyen, J. J.; Moreno, M. A. and Gogorcena, Y. 2013. Physiological, biochemical and molecular responses in four Prunus rootstocks submitted to drought stress. Tree Physiology. 33:1061-1075.

Kafkafi, U. 2008. Functions of the root system. In: Raviv, J. R. M. and Lieth H. (Eds). Soilless Culture: Theory and Practice. Elsevier, London WC1X 8RR, UK. 587 p.

Lal, A. M.; Kathpalia R.; Sisodia, R. and Shakya, R. 2018. Biotic Stress. 1045-1111 pp. In: Bhatla C. S. and Lal A. M. (Eds). Plant Physiology, Development and Metabolism. Springer. $1250 \mathrm{p}$.

Larsen, M.; Santner, J., Oburger, E., Wenzel, W. W. and Glud, N. R. 2015. $\mathrm{O}_{2}$ dynamics in the rhizosphere of young rice plants (Oryza sativa L.) as studied by planar optodes. Plant Soil 390:279-292.

Liao, C. T. and Lin, C. H. 2001. Physiological adaptation of crop plants to flooding stress. Proceedings of the National Science Council, Republic of China. Part B, Life sciences, 25(3):148-157.

Maestre, V. J. F. and Martínez, A. V. 2010. Effects of drip irrigation systems on the recovery of dissolved oxygen from hypoxic water. Science Direct. Agricultural Water Management. 97:1806-1812.

Makita, N.; Hirano, Y.; Sugimoto, T.; Tanikawa, T. and Ishii, H. 2015. Intraspecific variation in fine root respiration and morphology in response to in situ soil nitrogen fertility in a 100year-old Chamaecyparis obtusa forest. Oecologia. 1-9 pp.

Massol, A. 2005. Nutrientes y gases: Oxígeno disuelto. En: Manual de ecología microbiana. Departamento de biología. Universidad de Puerto Rico. Recinto universitario de Mayagüez.

Mazuela, P. 2010. Effect of oxygen supply on water uptake in a melon crop under soilless culture. Interciencia. 35(10):769-771.

M'Bou, A. T.; Saint-André, L.; de Grandcourt, A.; Nouvellon, Y.; Jourdan, C.; Mialoundama, F. and Epron, D. 2010. Growth and maintenance respiration of roots of clonal Eucalyptus cuttings: scaling to stand-level. Plant and Soil. 332(1-2):41-53.

Midmore, D. J.; Bhattarai, S. P.; Pendergast, L. and Torabi, M. 2007. Oxygation: aeration of subsurface drip irrigation water and its advantages for crop production, paper presented at the ANCID Conference 2007, Bundaberg, Australia, 19-22 August 2007.

Mohamed, M. S.; Abuarab, E. M. and Magdy, E. 2014. Root aeration improves yield and water use efficiency of irrigated potato in sandy clay loam soil. International Journal of Advanced Research. 2(10):310-320.

Morard, P.; Lacoste, L. and Silvestre, J. 1995. Effect of oxygen deficiency on uptake of water and mineral nutrients by tomato plants in soilless culture. J. Plant Nutr. 23:1063-1078. 
Murad, A.; Peñaranda, F.; Cárdenas, J.; Piragauta, M. y Relles, J. 2005. Oxígeno disuelto. Calidad del agua, Proyecto Fluoreciencia, Facultad del medio ambiente y recursos naturales, Universidad Distrital Francisco José Caldas. Bogotá, Colombia.

Papke, C.; Ramírez-Aguilar, C. and Antonio, C. 2014. Oxygen consumption under hypoxic conditions. 185-208 pp. In: van Dongen T. J. and Licausi, F. (Eds). Low-oxygen stress in plants. Oxygen sensing and adaptive responses to hypoxia. Plant Cell Monographs. Springer. $419 \mathrm{p}$.

Peverill, K. 1999. Soil Analysis: An interpretation manual. CSIRO Publishing, Australia. 208 p.

Porterfield, D. M. and Musgrave, M. E. 1998. The tropic response of plant root to oxygen: oxitropism in Pisum Sativum L. Planta. 206:1-6.

Rachmilevitch, S.; Lambers, H. and Huang, B. 2006. Root respiratory characteristics associated with plant adaptation to high soil temperature for geothermal and turf-type agrostis species. J. Exp. Botany. 57(3):623-631.

Raviv, M.; Wallach, R. and Blom, T. J. 2004. The effect of physical properties of soilless media on plant perfomance. A review. Acta Hortic. 644:251-259.

Rengasamy, P. 2000. Subsoil constraints and agricultural productivity. J. Indian Soil Sci. Soc. 48:674-682.

Schröder, F, G. and Lieth, J. H. 2004. Gas composition and oxygen supply in the root environment of substrates in closed hydroponic systems. Acta Hortic. 644:299-305.

Shi, K.; Hu, W. H.; Dong, D. K.; Zhou, Y. H. and Yu, J. Q. 2007. Low $\mathrm{O}_{2}$ supply is involved in the poor growth in root-restricted plants of tomato (Lycopersicon esculentum Mill.) Environ. Exp. Bot. 61:181-189.

Stepanova, A. U.; Polyakova, L.; Dolgikh, I.; Yu, I. and Vartapetian, B. B. 2002. The response of sugarcane (Saccharum officinarum) cultured cells to anoxia and the selection of a tolerant cell line. Russian Journal of Plant Physiology. 49:406-412.

Toro, G. and Pint, M. 2015. Plant respiration under low oxygen. Chil. J. of Agr. Res. 75:57-70.

Urrestarazu, M. and Mazuela, P. C. 2005. Effect of slow-release oxygen supply by fertigation on horticultural crops under soilless culture. Sci. Hort. 106:484-490.

Urrestarazu, M.; Mazuela P.; Ventura, F. y Guillén, C. 2006. Beneficios de la aplicación de oxígeno en cultivos sin suelo. Agrícola Vergel: fruticultura, horticultura, floricultura Vida rural 292:195-200.

van Dongen, T. J. and Licausi, F. 2015. Oxygen sensing and signaling. Ann. Rev. Plant Biol. 66:345-367.

van Dongen, T. J.; Gupta, K. J.; Ramírez-Aguilar, S. J.; Araujo, W. L.; Nunes-Nesi, A. and Fernie, A. R. 2011. Regulation of respiration in plants: a role for alternative metabolic pathways. J. Plant Physiology. 168(12):1434-1443. 